\title{
Collisions of supersonic clouds
}

\author{
Andrew McLeod ${ }^{1,2}$, Jan Palouš ${ }^{1}$ and Anthony Whitworth ${ }^{2}$ \\ ${ }^{1}$ Astronomical Institute, Academy of Sciences of the Czech Republic \\ ${ }^{2}$ Dept. of Physics and Astronomy, Cardiff University, \\ email: Andrew.McLeod@astro.cf.ac.uk
}

\begin{abstract}
We present simulations of supersonic collisions between molecular clouds of mass $500 \mathrm{M}_{\odot}$ and radius $2.24 \mathrm{pc}$. The simulations are performed with the SEREN SPH code. The code treats the energy equation and the associated transport of heating and cooling radiation. The formation of protostars is captured by introducing sink particles. Low velocity collisions form a shock-compressed layer which fragments to form stars. For high-velocity collisions, $v_{r e l} \gtrsim$ $5 \mathrm{~km} \mathrm{~s}^{-1}$, the non-linear thin shell instability strongly disrupts the shock-compressed layer, and may inhibit the formation of stars.
\end{abstract}

Keywords. instabilities, shock waves, ISM:clouds, stars: formation, stars: mass function

\section{Introduction}

Cloud-cloud collisions may be important in understanding the star-formation process. The supersonic collision of clouds can compress gas causing it to become gravitationally unstable and collapse into stars. Clouds may also merge or disperse in collisions. Feedback from stellar clusters created by cloud-cloud collisions may cause the recoil of the original clouds, which can then go on to collide with other clouds.

Whitworth et al. (1994) analysed the effects of the gravitational instability in a shocked layer. They found that fragments in the layer could be Jeans-unstable and collapse gravitationally, with the fastest modes being those of approximately the Jeans length. They derive the typical fragment separation

$$
L_{\text {fragment }} \sim c_{s} \times t_{\text {fragment }} \sim \mathcal{M}^{1 / 2} \times \frac{c_{s}}{\left(G \bar{\rho}_{\text {layer }}\right)^{1 / 2}},
$$

where $\mathcal{M}$ is the Mach number of the collision relative to the sound speed in the layer $c_{s}$.

Vishniac (1994) describes for the first time the non-linear thin shell instability (NTSI). He studies the growth of the instability in an initially perturbed shock-compressed slab. Ram pressure acts along the collision axis, however thermal pressure is always normal to the slab. In the NTSI, this creates a shear in the outer layers of a perturbed slab, driving material towards the extremities of the perturbation and strengthening it.

Vishniac derives the growth rate of the NTSI as

$$
\tau^{-1}(k) \propto k^{3 / 2} \quad \text { over unstable wavenumbers } \quad L^{-1}>k \gg\left(\frac{V_{s}}{c_{s}}\right) R^{-1},
$$

where $L$ is the thickness of the slab, $V_{s}$ is the velocity of the shock, $c_{s}$ is the sound speed in the shock, and $R$ is the propagation distance over which zeroth order shock properties evolve. For a stationary slab, $R=V_{e} \times t$, the velocity of the external gas multiplied by the time since the slab was formed. The NTSI should be strongest for high Mach number collisions, as these will have a higher density contrast. 




Figure 1. Projected densities $\left(\mathrm{g} \mathrm{cm}^{-3}\right)$ in the $\mathrm{y}^{-\mathrm{z}}$ plane. The solid white line indicates the expected length scale of the gravitational instability. Solid black dots indicate the presence of a sink particle (not to scale). From top left to bottom right: Mach 4, 8, 12 and 16.

\section{Method}

We set up spheres of radius $2.24 \mathrm{pc}$ and mass $500 \mathrm{M}_{\odot}$ containing six million settled SPH particles. The spheres are initially touching. We collide our clouds head-on at Mach 4, 8, 12, 16 and 40, assuming a sound speed of $0.25 \mathrm{~km} \mathrm{~s}^{-1}$. We also superimpose a weak turbulent velocity field of purely solenoidal turbulence with r.m.s. velocity $0.1 \mathrm{~km} \mathrm{~s}^{-1}$. This is too weak to significantly support the cloud but may seed instabilities.

We use an MPI-parallelized version of the SEREN (Hubber et al., 2010) code to simulate cloud-cloud collisions with smoothed particle hydrodynamics (SPH). We include self-gravity. We use sink particles to represent protostellar objects with a sink creation density of $10^{-12} \mathrm{~g} \mathrm{~cm}^{-3}$. We use the Monaghan (1997) method of artificial viscosity; we use the Morris \& Monaghan (1997) switch to reduce viscosity away from shocks. As in Price \& Federrath (2010), we use $\beta=4$ instead of the standard $\beta=2$ to enhance viscosity in high Mach number shocks.

We use the Stamatellos et al. (1997) method to follow the energy equation and capture the effects of heating and cooling radiation. One limitation of this method is that it assumes the gas and dust are well-coupled and so below a density of $\sim 10^{5} \mathrm{~cm}^{-3}$ it is likely to overestimate the cooling and underestimate the gas temperature.

\section{Results}

\subsection{Low velocity collisions (Mach 4, 8, 12 \& 16)}

Figure 1 shows column densities along the collision axis. In the Mach 4 and Mach 8 collisions the layer appears to have fragmented into a filamentary structure. For the Mach 12 and Mach 16 collisions the network of linear filaments has been largely replaced by a more 'clumpy' structure. Although this is also organized into a filamentary network, in many cases clumps exist that are completely separate from these networks. We suggest that this is due to the combination of the gravitational instability and the NTSI as the Mach number increases.

The expected length scale of fragmentation is larger than that observed in the filamentary structure by approximately a factor of two. The exception to this is the slowest 
Sink mass function
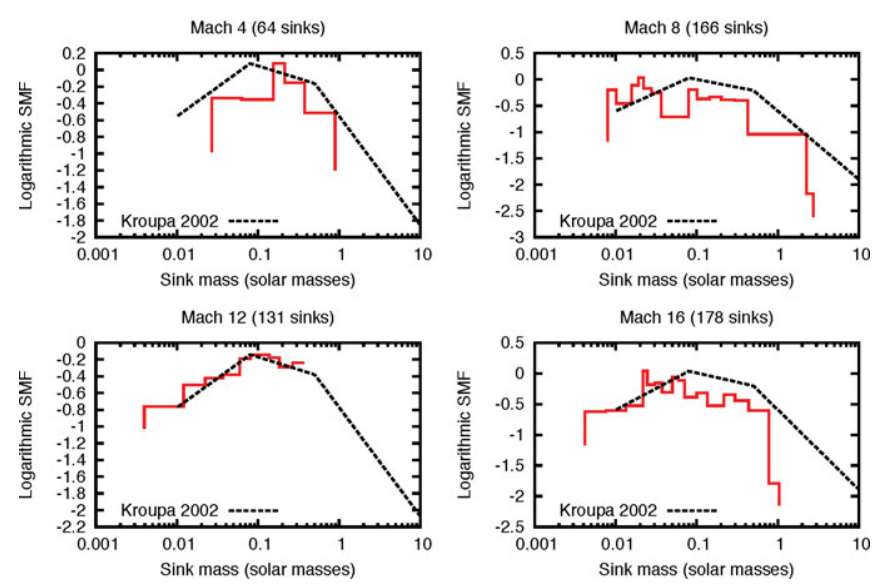

Figure 2. Sink mass function at end of simulation. Each bin contains an equal number of sinks. The Kroupa (2002) IMF is overlaid, fitted to match the highest bin.

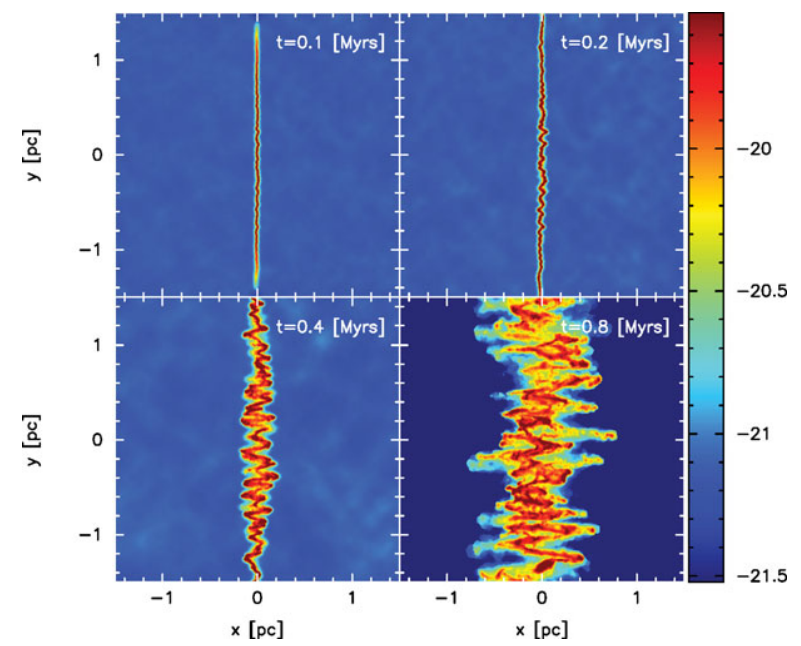

Figure 3. Cross-sections of density $\left(\mathrm{g} \mathrm{cm}^{-3}\right)$ in the $\mathrm{x}-\mathrm{y}$ plane of the Mach 40 cloud-cloud collision.

(Mach 4) collision, but this snapshot is taken at a later time when the pattern of fragments has begun to collapse.

All four low-velocity simulations produce sink particles. Figure 2 shows the sink mass functions; these are noisy but compatible with the Kroupa (2002) IMF.

\subsection{High velocity collision (Mach 40)}

This collision produces no sink particles. Instead, the shocked layer is unstable to the NTSI and eventually breaks up completely. This can be seen in figure 3 which shows cross-sections of density in the $\mathrm{x}-\mathrm{y}$ plane. The initially weak perturbations grow nonlinearly until they saturate and the layer becomes bloated and chaotic. This collision does not form stars.

In figure 4 we show the growth rates of the NTSI as a function of wavenumber $|\boldsymbol{k}|$, for a number of time intervals. At early times the NTSI is not fully developed, but at intermediate times the growth rate obeys the expected relation $\tau^{-1} \propto|\boldsymbol{k}|^{3 / 2}$ up to the 


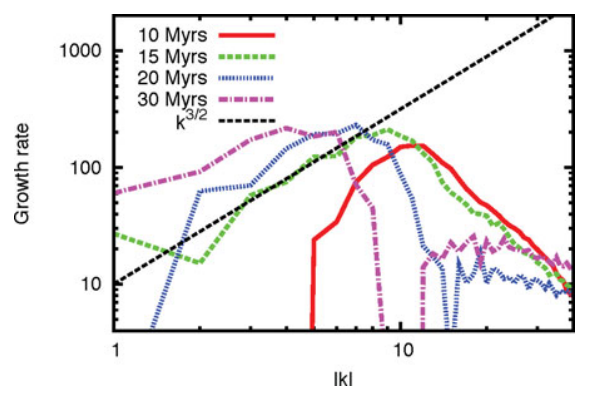

Figure 4. Growth rates of the NTSI as a function of wavenumber $|\boldsymbol{k}|$ at different times. The dashed black line shows the solution of Vishniac, $\tau^{-1} \propto|\boldsymbol{k}|^{3 / 2}$.

wavenumber equivalent to the thickness of the layer $|\boldsymbol{k}| \sim 10$. At later times than shown the NTSI is saturated and no longer obeys the Vishniac growth rate.

\section{Conclusions}

We have simulated head-on cloud-cloud collisions of $500 \mathrm{M}_{\odot}$ clouds with uniform density and radius $2.24 \mathrm{pc}$. We have simulated collision velocities of Mach 4, 8, 12, 16 and 40. All these collisions produce a shock-compressed layer. The lower velocity collisions form a network of filaments. Stars form in the densest parts of these filaments. Intermediate velocity collisions are affected by the non-linear thin shell instability, causing the filamentary structure in the layer to be overlaid with a more clumpy texture. Stars then form out of these filaments and clumps. The highest velocity collision produces a layer which is strongly affected by the NTSI and does not form stars. We reproduce the expected growth rate of the NTSI for its early evolution.

Plots of SPH data in this work were produced with SPLASH, written by Daniel Price (2007). This research has made use of NASA's Astrophysics Data System. Simulations were performed using the Cardiff University ARCCA (Advanced Research Computing CArdiff) cluster (MERLIN). Andrew McLeod is funded as an Early-Stage Researcher by the EC-funded CONSTELLATION Marie Curie training network MRTN-CT-2006035890 and has an STFC Studentship at Cardiff University in abeyance.

\section{References}

Bate, M., Bonnell, I., \& Price, N. 1995 MNRAS, 277, 362

Barnes, J. \& Hut, P. 1986 Nature, 324, 446

Hubber, D. A., Batty, C. P., McLeod, A., \& Whitworth, A. P. 2010, A\&A, submitted

Kroupa, P. 2002 Science, 295, 82

Monaghan, J. 1997 JCoPh, 136, 298

Morris, J. \& Monaghan, J. 1997 JCoPh, 136, 41

Price, D. 2007, PASA, 24, 159

Price, D. \& Federrath, C. 2010 MNRAS, MNRAS, 406, 1659

Stamatellos, D., Whitworth, A., Bisbas, T., \& Goodwin, S. 2007 MNRAS, 475, 37

Vishniac, E. T. 1994, ApJ, 428, 186

Whitworth, A., Bhattal, A., Chapman, S., Disney, M., \& Turner, J. 1994 AËA, 290, 421 